\title{
A new extended Reynolds equation for gas bearing lubrication based on the method of moments
}

\author{
Xiao-Jun Gu ${ }^{1}$ Haijun Zhang ${ }^{2}$ David R. Emerson ${ }^{1}$
}

Received: 16 July 2015 / Accepted: 23 November 2015 / Published online: 7 January 2016

(c) The Author(s) 2016. This article is published with open access at Springerlink.com

\begin{abstract}
An extended Reynolds equation based on the regularised 13 moment equations and lubrication theory is derived for gas slider bearings operating in the early to upper transition regime. The new formulation performs well beyond the capability of the conventional Reynolds equation modified with simple velocity-slip models. Both load capacity and pressure distribution can be reliably predicted by the extended Reynolds equation and are in good agreement with available direct simulation Monte Carlo data. In addition, the equations are able to provide both velocity and stress information, which is not conveniently recovered from available kinetic models. Tests indicate that the equations can provide accurate data for Knudsen numbers up to unity but, as expected, begin to deteriorate afterwards.
\end{abstract}

Keywords Reynolds equation - Lubrication theory · Slider bearing $\cdot$ Moment method $\cdot$ Knudsen number . Non-equilibrium

\section{Introduction}

In modern computer hard disk drives, the gas between the read-write head and the surface of a spinning disk forms an air slider bearing that supports the head floating above the disk. Traditionally, the classical Reynolds lubrication

Xiao-Jun Gu

xiaojun.gu@stfc.ac.uk

1 Scientific Computing Department, STFC Daresbury Laboratory, Warrington WA4 4AD, UK

2 College of Mechanical-Electrical Engineering, Jiaxing University, Jiaxing 314001, Zhejiang, China equation has been used to model slider bearings. In the theory of lubrication, the continuum description is assumed and inertial terms are negligible (Reynolds 1886). A pressure equation can then be obtained with one dimension less than the Navier-Stokes (NS) equations. However, as modern read-write heads are typically floating $50 \mathrm{~nm}$ or less above the moving disk, the characteristic length scale involved is comparable to the molecular mean free path, $\lambda$, and the continuum assumption is no longer considered to be valid. Any gas in such a narrow spacing will suffer from significant non-equilibrium effects, which can be measured by the Knudsen number, $K n$, and relates the ratio of the mean free path, $\lambda$, to the characteristic length scale of the device. For convenience, it is possible to classify four distinct flow regimes based on the Knudsen number (Barber and Emerson 2006): (1) $K n<10^{-3}$, represents the continuum flow regime where no-slip boundary conditions can generally be applied; (2) $10^{-3}<K n<10^{-1}$, indicates that the flow is in the slip regime and boundary conditions need to account for velocity-slip and temperature-jump conditions; (3) $10^{-1}<K n<10$, represents the transition flow regime and results obtained using continuum-based equations are no longer considered reliable; and (4) $K n>10$, is the free molecular or collisionless flow regime and the continuum hypothesis is not valid.

In the case of slider bearings operating in the slip-flow regime, it is usually adequate to modify the Reynolds equation with a velocity-slip boundary condition (Burgdorfer 1959; Hsia and Domoto 1983; Mitsuya 1993; Bahukudumbi and Beskok 2003; Chen and Bogy 2010). However, for many problems, especially those related to disk-drive heads, the operating conditions lie well within the transition regime. This is a region where non-equilibrium effects come not only from the wall but also from the Knudsen layer (Cercignani 2000; Lilley and Sader 2008; Gu et al. 
2010). Modification of the slip boundary condition alone is not sufficient to compensate for the substantial deviation from the continuum assumption. The Reynolds lubrication equation with a first-order velocity-slip boundary condition will over-predict the pressure rise (Burgdorfer 1959), while a second-order boundary condition consistently under-predicts the pressure rise (Hsia and Domoto 1983). To account for the non-equilibrium effects from the wall and the Knudsen layer, Fukui and Kaneko (1988) derived a generalised lubrication equation (FK model) for arbitrary Knudsen number based on the linearised Boltzmann equation with the Bhatnagar-Gross-Krook (BGK) collision model (Bhatnagar et al. 1954). Their derivation focused on the Poiseuille flow rate and no analytical expressions for the velocity profile and shear stress were given, although they can be recovered from the solution of the pressure equation. Due to the complexity of the original expression of the FK model, a piecewise curve fit formula was provided for its practical use (Fukui and Kaneko 1990). However, Cercignani et al. (2004) pointed out that the database given by Fukui and Kaneko (1990) is inaccurate. Furthermore, as noted by Chen and Bogy (2010), there is a contact pressure singularity in the FK model. Cercignani et al. (2007) derived a Reynolds equation on the basis of the BGK and ellipsoidal statistical BGK Boltzmann equation. Its solution of pressure requires an accurate evaluation of the Abramowitz functions (Abramowitz and Stegun 1970), and their results show that the ellipsoidal statistical BGK model slightly under-predicts the pressure rise compared to the BGK model. In both kinetic approaches, an accurate solution can be obtained from the linearised Boltzmann equation, but each requires significant effort and diligent programming.

To study the physics of gas slider bearings in the transition regime, the direct simulation Monte Carlo (DSMC) approach has generally been employed to simulate the flow details (Alexander et al. 1994; Liu and Ng 2001; Jiang et al. 2005; John and Damodaran 2009). Gas velocity profiles between the disk and slider, the shear stress on the wall, as well as the pressure distribution, can be obtained by averaging particle movement statistically. Data obtained using DSMC provide an accurate and reliable way to validate our lubrication theory in the transition regime. However, the method is computationally expensive and is generally inappropriate for engineering design and analysis. A lubrication equation that can capture Knudsen layer effects offers great potential for design engineers. In the present study, we have used the method of moments to derive an extended Reynolds equation that can be used in the transition regime and can provide design information beyond the FK pressure distribution and without substantial effort.

The method of moments (Grad 1949) provides an approximation to the Boltzmann equation. In this approach, the Boltzmann equation is satisfied in a certain average sense rather at the molecular level. Macroscopic quantities, such as pressure, $p$, gas velocity, $u_{i}$, stress, $\sigma_{i j}$, and heat flux, $q_{i}$, are moments of the molecular distribution function. As the constitutive relationships for $\sigma_{i j}$ and $q_{i}$ in the NavierStokes-Fourier (NSF) equations are no longer valid in the transition regime, governing equations for $\sigma_{i j}$ and $q_{i}$ are used, which can be derived from the Boltzmann equation. However, the fluxes of $\sigma_{i j}$ and $q_{i}$ appear in a set of governing equations, which are necessary to close the system of equations. In the original approach of the moment method, the fluxes of $\sigma_{i j}$ and $q_{i}$ are calculated from a truncated distribution function of Hermite polynomials. The resultant set of equations is referred to as Grad's 13 moment equations (G13) (Grad 1949). Applying a Chapman-Enskoglike expansion, a set of regularised 13 moment equations (R13) was developed (Struchtrup and Torrilhon 2003), which significantly improves the capability of the G13 equations, particularly in confined geometries. The regularisation procedure can be readily extended to incorporate higher moments such as the R26 equations ( $\mathrm{Gu}$ and Emerson 2009). Recently, a velocity-slip boundary condition constructed from the R13 solution of a half-space problem was adopted to modify the traditional NS-based Reynolds equation (Yang, Zhang and Liu 2015). In the present work, the extended Reynolds equation is derived from kinetic theory and the R13 equations, rather than the NS equations.

In small-scale devices, such as micro-electro-mechanical systems (MEMS), Reynolds numbers are usually very small. Consequently, a linearised set of moment equations is generally adequate to capture the necessary flow features for many practical purposes. Macroscopic quantities in the Knudsen layer can be expressed as superpositions of exponentials of different widths obtained from the linearised moment equations (Struchtrup 2008; Gu et al. 2010; Gu and Emerson 2014). The linearised R26 equations have been shown to provide a more accurate description of the Knudsen layer than the R13 equations. However, the linearised R13 equations are adopted in the present study to derive an extended Reynolds equation because of their relative simplicity. As the pressure variation in the bearing system is significant, it is inappropriate to linearise the pressure-related terms for thin-film gas flow between two plates. To maintain the accuracy of the R13 equations, the pressure-related nonlinear terms need to be retained. In this way, the R13 equations are semi-linearised. Indeed, we will show that this new system of equations achieves a good level of accuracy and is suitable for engineering design and analysis.

In the next section, the air bearing problem is specified and the semi-linearised R13 equations are presented. We will then apply lubrication theory to the moment equations to reduce the complexity of the equation set. Using 
this approximation, the solutions derived from the extended Reynolds equations are presented and are compared with available DSMC data (Alexander et al. 1994; Liu and Ng 2001; $\mathrm{Ng}$ et al. 2002).

\section{Problem specification and mathematical formulation}

A schematic of a three-dimensional slider bearing geometry sitting in a Cartesian coordinate system, $x_{i}=[x, y, z]$, is shown in Fig. 1. The bottom plate, located at $z=0$, moves with a constant velocity, $u_{w}$, in the $x$-direction and has a length, $L$, and a width, $W$. The upper plate is fixed and has a constant pitch angle, $\theta$. The gap between the plates in the $z$-direction, $H$, varies and has a minimum value of $H_{0}$ at the end of the bearing. The gap is much smaller than the length and width of the plates so that $\varepsilon=H_{0} / L \ll 1$, $H_{0} / W \ll 1$, with $\sin \theta \simeq 0$ and $\cos \theta \simeq 1$. The gas flow between the plates is considered to be isothermal and at a constant temperature, $T$. Outside the domain of the slider bearing, the pressure is at atmospheric conditions, $p_{0}$, and the mean free path of gas molecules, $\lambda$, can be estimated by

$\lambda=\frac{\mu}{p_{0}} \sqrt{\frac{\pi R T}{2}}$,

where $\mu$ is the gas viscosity and $R$ the gas constant, respectively. Based on the minimum gap height, $H_{0}$, the Knudsen number at the exit, $K n$, is defined by

$K n=\frac{\lambda}{H_{0}}$.

It is further assumed that the gas obeys the ideal gas law, $p=\rho R T$.

The gas flow inside the slider bearing obeys the mass conservation law:

$\frac{\partial \rho}{\partial t}+\frac{\partial \rho u_{i}}{\partial x_{i}}=0$,

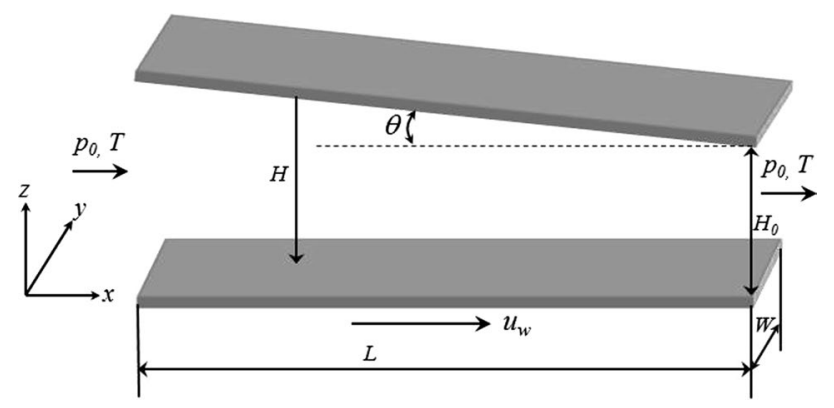

Fig. 1 Schematic of a three-dimensional slider geometry where $\rho$ is the density of the gas and $u_{i}=[u, v, w]$ is the gas velocity. The momentum equation is required to describe the gas movement. In terms of stress, $\sigma_{i j}$, and pressure, $p$, the momentum equation reads

$\frac{\partial \rho u_{i}}{\partial t}+\frac{\partial \rho u_{i} u_{k}}{\partial x_{k}}+\frac{\partial p}{\partial x_{i}}+\frac{\partial \sigma_{i k}}{\partial x_{k}}=0$.

Instead of using a constitutive relationship for velocity and stress to close Eqs. (3) and (4), the governing equations for the stress, $\sigma_{i j}$, and the heat flux, $q_{i}$, are derived from the moments of the Boltzmann equation to capture any nonequilibrium effects. The semi-linearised equations of $\sigma_{i j}$ and $q_{i}$ for low-speed isothermal flows, in terms of primitive variables, are:

$\frac{\partial \sigma_{i j}}{\partial t}+\frac{\partial m_{i j k}}{\partial x_{k}}+\frac{4}{5} \frac{\partial q_{<i}}{\partial x_{j>}}+2 p \frac{\partial u_{<i}}{\partial x_{j>}}=\underline{-\frac{p}{\mu} \sigma_{i j}}$,

and

$\frac{\partial q_{i}}{\partial t}+\frac{1}{2} \frac{\partial R_{i k}}{\partial x_{k}}+\frac{1}{6} \frac{\partial \Delta}{\partial x_{i}}+R T \frac{\partial \sigma_{i k}}{\partial x_{k}}=-\frac{2}{3} \frac{p}{\mu} q_{i}$,

in which the linear term of the higher moments $m_{i j k}, R_{i j}$, and $\Delta$ for Maxwell molecules (MM) is approximated by (Struchtrup 2005, 2008):

$m_{i j k}=-2 \frac{R T \mu}{p} \frac{\partial \sigma_{<i j}}{\partial x_{k>}}$,

$R_{i j}=-\frac{24}{5} \frac{R T \mu}{p} \frac{\partial q_{<i}}{\partial x_{j>}}=-4.8 \frac{R T \mu}{p} \frac{\partial q_{<i}}{\partial x_{j>}}$

and

$\Delta=-12 \frac{R T \mu}{p} \frac{\partial q_{k}}{\partial x_{k}}$.

Equations (3)-(9) constitute a semi-linearised set of regularised 13 moment equations where the underlined terms in Eqs. (5) and (6) indicate the pressure-related nonlinear components.

Recently, Gupta and Torrilhon (2012) calculated the hard sphere (HS) collision coefficients for the R13 moment equation. The only difference between HS and MM collision models for the R13 moment equation is the approximation for the higher moment, $R_{i j}$. Instead of Eq. (8), the expression for $R_{i j}$ for the HS model is:

$R_{i j}^{\mathrm{HS}}=-\frac{4704}{1235} \frac{R T \mu}{p} \frac{\partial q_{<i}}{\partial x_{j>}} \approx-3.8 \frac{R T \mu}{p} \frac{\partial q_{<i}}{\partial x_{j>}}$.

Comparison of Eqs. (8) and (10) shows that the value of the gradient coefficient is reduced from 4.8, for MM, to 3.8 for the HS model. The remaining coefficients for the other moments of MM and HS models are identical. As a consequence, both MM and HS models produce results that are 
similar, which will be shown in Sect. 4. For this paper, the coefficients of the MM model are employed to derive the Reynolds equation for the slider bearing.

\section{Lubrication approximation and extended Reynolds equation}

Lubrication theory is an approximate description of flows at low Reynolds numbers through narrow geometries with slow changes in curvature. Under the condition that $\varepsilon=H_{0} / L \ll 1$, lubrication theory scales the variables in the following way:
By using lubrication theory and neglecting all terms of $O\left(\varepsilon^{2}\right)$ and higher, the momentum equations can be simplified significantly. In particular, Eq. (14) indicates that the pressure gradient in the $z$-direction can be approximated as zero so that the pressure can be regarded as a two-dimensional variable. For convenience and ready comparison with available kinetic data, it is written as:

$P=P(\bar{x}, \bar{y})=\frac{p}{p_{0}}=\frac{\bar{p}}{\varepsilon}$.

Without the terms of $O\left(\varepsilon^{2}\right)$ and higher, Eqs. (12)-(18) can be grouped into two independent sets: one for flow in the

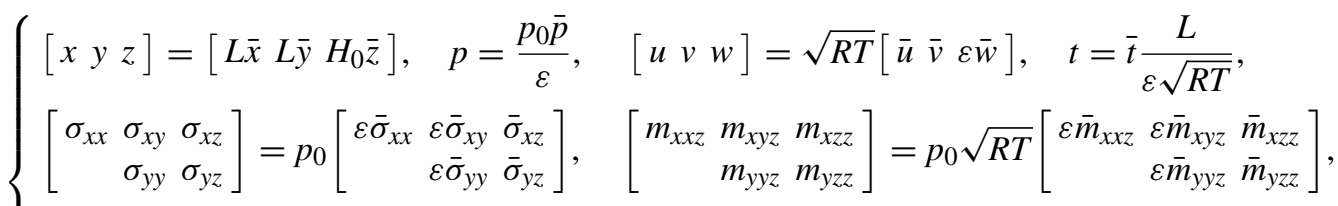

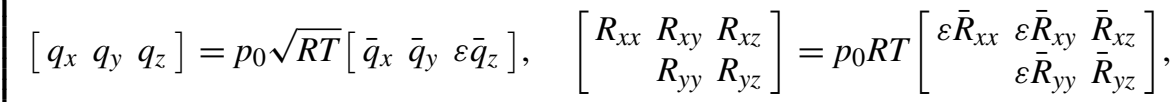

$$
\begin{aligned}
& \Delta=p_{0} R T \bar{\Delta}, H=H_{0} h \text {. }
\end{aligned}
$$

Inserting Eq. (11) into Eqs. (4)-(6) and neglecting inertial terms in the momentum equations under lubrication theory, the resulting momentum equations for a three-dimensional thin gas flow are expressed in non-dimensional form as:

$$
\begin{aligned}
& \varepsilon^{2}\left(\frac{\partial \bar{\sigma}_{x x}}{\partial \bar{x}}+\frac{\partial \bar{\sigma}_{x y}}{\partial \bar{y}}\right)+\frac{\partial \bar{p}}{\partial \bar{x}}+\frac{\partial \bar{\sigma}_{x z}}{\partial \bar{z}}=0, \\
& \varepsilon^{2}\left(\frac{\partial \bar{\sigma}_{x y}}{\partial \bar{x}}+\frac{\partial \bar{\sigma}_{y y}}{\partial \bar{y}}\right)+\frac{\partial \bar{p}}{\partial \bar{y}}+\frac{\partial \bar{\sigma}_{y z}}{\partial \bar{z}}=0,
\end{aligned}
$$

and

$\varepsilon^{2}\left(\frac{\partial \bar{\sigma}_{x z}}{\partial \bar{x}}+\frac{\partial \bar{\sigma}_{y z}}{\partial \bar{y}}-\frac{\partial \bar{\sigma}_{x x}}{\partial \bar{z}}-\frac{\partial \bar{\sigma}_{y y}}{\partial \bar{z}}\right)+\frac{\partial \bar{p}}{\partial \bar{z}}=0$.

The relationship, $\bar{\sigma}_{x x}+\bar{\sigma}_{y y}+\bar{\sigma}_{z z}=0$, has been used in Eq. (14). The equations for $\sigma_{i j}$ and $q_{i}$ in scaled non-dimensional form in Cartesian coordinates are written as: $x$-direction, the other in the $y$-direction. For flow in the $x$-direction, they are:

$\varepsilon \frac{\partial P}{\partial \bar{x}}+\frac{\partial \bar{\sigma}_{x z}}{\partial \bar{z}}=0$,

$\frac{\partial \bar{m}_{x z z}}{\partial \bar{z}}+\frac{2}{5} \frac{\partial \bar{q}_{x}}{\partial \bar{z}}+P \underline{\partial \frac{\partial \bar{u}}{\partial \bar{z}}}=\underline{-P \frac{\bar{\sigma}_{x z}}{K_{O}}}$,

$\frac{1}{2} \frac{\partial \bar{R}_{x z}}{\partial \bar{z}}+\frac{\partial \bar{\sigma}_{x z}}{\partial \bar{z}}=-\frac{2 P}{3} \frac{\bar{q}_{x}}{K_{O}}$,

$\bar{m}_{x z z}=-\frac{16}{15} \frac{K_{o}}{P} \frac{\partial \bar{\sigma}_{x z}}{\partial \bar{z}}$

and

$\varepsilon^{3}\left(\frac{\partial \bar{\sigma}_{x z}}{\partial \bar{t}}+\frac{\partial \bar{m}_{x x z}}{\partial \bar{x}}+\frac{\partial \bar{m}_{x y z}}{\partial \bar{y}}+\frac{2}{5} \frac{\partial \bar{q}_{z}}{\partial \bar{x}}\right)+\varepsilon^{2} \bar{p} \frac{\partial \bar{w}}{\partial \bar{x}}+\varepsilon\left(\frac{\partial \bar{m}_{x z z}}{\partial \bar{z}}+\frac{2}{5} \frac{\partial \bar{q}_{x}}{\partial \bar{z}}\right)+\bar{p} \frac{\partial \bar{u}}{\partial \bar{z}}=-\sqrt{\frac{\pi}{2}} \frac{H_{0} \bar{p}}{\lambda} \bar{\sigma}_{x z}$,

$\varepsilon^{3}\left(\frac{\partial \bar{\sigma}_{y z}}{\partial \bar{t}}+\frac{\partial \bar{m}_{x y z}}{\partial \bar{x}}+\frac{\partial \bar{m}_{y y z}}{\partial \bar{y}}+\frac{2}{5} \frac{\partial \bar{q}_{z}}{\partial \bar{y}}\right)+\varepsilon^{2} \bar{p} \frac{\partial \bar{w}}{\partial \bar{y}}+\varepsilon\left(\frac{\partial m_{y z z}}{\partial \bar{z}}+\frac{2}{5} \frac{\partial q_{y}}{\partial \bar{z}}\right)+\bar{p} \frac{\partial \bar{v}}{\partial \bar{z}}=-\sqrt{\frac{\pi}{2}} \frac{H_{0} \bar{p}}{\lambda} \bar{\sigma}_{y z}$,

$\varepsilon^{3}\left(\frac{\partial \bar{q}_{x}}{\partial \bar{t}}+\frac{1}{2} \frac{\partial \bar{R}_{x x}}{\partial \bar{x}}+\frac{1}{2} \frac{\partial \bar{R}_{x y}}{\partial \bar{y}}+\frac{\partial \bar{\sigma}_{x x}}{\partial \bar{x}}+\frac{\partial \bar{\sigma}_{x y}}{\partial \bar{y}}\right)+\frac{\varepsilon^{2}}{6} \frac{\partial \bar{\Delta}}{\partial \bar{x}}+\varepsilon\left(\frac{1}{2} \frac{\partial \bar{R}_{x z}}{\partial \bar{z}}+\frac{\partial \bar{\sigma}_{x z}}{\partial \bar{z}}\right)=-\frac{2}{3} \sqrt{\frac{\pi}{2}} \frac{H_{0} \bar{p}}{\lambda} \bar{q}_{x}$

and

$\varepsilon^{3}\left(\frac{\partial \bar{q}_{y}}{\partial \bar{t}}+\frac{1}{2} \frac{\partial \bar{R}_{x y}}{\partial \bar{x}}+\frac{1}{2} \frac{\partial \bar{R}_{y y}}{\partial \bar{y}}+\frac{\partial \bar{\sigma}_{x y}}{\partial \bar{x}}+\frac{\partial \bar{\sigma}_{y y}}{\partial \bar{y}}\right)+\frac{\varepsilon^{2}}{6} \frac{\partial \bar{\Delta}}{\partial \bar{y}}+\varepsilon\left(\frac{1}{2} \frac{\partial \bar{R}_{y z}}{\partial \bar{z}}+\frac{\partial \bar{\sigma}_{y z}}{\partial \bar{z}}\right)=-\frac{2}{3} \sqrt{\frac{\pi}{2}} \frac{H_{0} \bar{p}}{\lambda} \bar{q}_{y}$. 


$$
\bar{R}_{x z}=-\frac{12}{5} \frac{K_{o}}{P} \frac{\partial \bar{q}_{x}}{\partial \bar{z}} .
$$

Similarly, the equations for flow in the $y$-direction can be written as,

$\varepsilon \frac{\partial P}{\partial \bar{y}}+\frac{\partial \bar{\sigma}_{y z}}{\partial \bar{z}}=0$,

$\frac{\partial \bar{m}_{y z z}}{\partial \bar{z}}+\frac{2}{5} \frac{\partial \bar{q}_{y}}{\partial \bar{z}}+P \underline{\partial \frac{\partial \bar{v}}{\partial \bar{z}}}=-P \frac{\bar{\sigma}_{y z}}{K_{o}}$,

$\frac{1}{2} \frac{\partial \bar{R}_{y z}}{\partial \bar{z}}+\frac{\partial \bar{\sigma}_{y z}}{\partial \bar{z}}=-\frac{2 P}{3} \frac{\bar{q}_{y}}{K_{o}}$,

$\bar{m}_{y z z}=-\frac{16}{15} \frac{K_{o}}{P} \frac{\partial \bar{\sigma}_{y z}}{\partial \bar{z}}$

and

$\bar{R}_{y z}=-\frac{12}{5} \frac{K_{o}}{P} \frac{\partial \bar{q}_{y}}{\partial \bar{z}}$,

in which

$K_{o}=\sqrt{\frac{2}{\pi}} K n$.

Equation (19) indicates that $P$ is independent of $\bar{z}$, and the underlined nonlinear terms in Eqs. (21), (22), (26) and (27) reduce to linear terms.

There were no explicit wall boundary conditions available for the moment equations, which hamper the use of the moment method in a wide range of practical applications, although the principle for constructing wall boundary conditions was suggested by Grad (1949). The first set of explicit wall boundary conditions (Gu and Emerson 2007) for the regularised moment equations is constructed from Maxwell's kinetic boundary condition (Maxwell 1879). The boundary conditions are further improved for the R13 and R26 moment equations (Torrilhon and Struchtrup 2008; Gu and Emerson 2009). For Eqs. (20)-(29), the linearised boundary conditions for a solid wall with normal vector, $n_{i}$, and tangential vector, $\tau_{i}$, are expressed by $(\mathrm{Gu}$ et al. 2009):

$\bar{u}_{\tau}=-\gamma \frac{\bar{\sigma}_{n \tau}}{P}-\frac{5 \bar{m}_{n n \tau}+2 \bar{q}_{\tau}}{10 P}$,

$\bar{q}_{\tau}=-\frac{5}{18} \gamma\left(7 \bar{\sigma}_{n \tau}+\bar{R}_{n \tau}\right)-\frac{5 \bar{u}_{\tau} P}{3}-\frac{10 \bar{m}_{n n \tau}}{9}$

and

$\bar{u}_{n}=0$, with

$\gamma=\frac{2-\alpha}{\alpha} \sqrt{\frac{\pi}{2}}$,

where $\alpha$ is the accommodation coefficient, which indicates that a fraction $(1-\alpha)$ of gas molecules will undergo "specular" reflection, while the remaining fraction $\alpha$ will be "diffusely" reflected with a Maxwellian distribution function at the wall temperature (Maxwell 1879). Here, $\bar{u}_{\tau}=\bar{u}_{i} \tau_{i}$ and $\bar{u}_{n}=\bar{u}_{i} n_{i}$, are the normalised tangential velocity slip at the wall and the velocity normal towards the wall, respectively, and $\bar{\sigma}_{n \tau}=\bar{\sigma}_{i j} n_{i} \tau_{j}, \bar{q}_{\tau}=\bar{q}_{i} \tau_{i}, \bar{m}_{n n \tau}=\bar{m}_{i j k} n_{i} n_{j} \tau_{k}$ and $\bar{R}_{n \tau}=\bar{R}_{i j} n_{i} \tau_{j}$ are the tangential components of $\bar{\sigma}_{i j}, \bar{q}_{i}, \bar{m}_{i j k}$, $\bar{R}_{i j}$ relative to the wall.

The solutions of Eqs. (20)-(24) are:

$\bar{q}_{x}=C_{1 x} e^{-a \bar{z}}+C_{2 x} e^{a \bar{z}}+\frac{1}{A_{q}} \frac{K_{o}}{P}\left(\varepsilon \frac{\partial P}{\partial \bar{x}}\right)$,

$\bar{\sigma}_{x z}=-\left(\varepsilon \frac{\partial P}{\partial \bar{x}}\right) \bar{z}+C_{3 x}$,

$\bar{u}=\frac{\bar{z}^{2}}{2 K_{o}}\left(\varepsilon \frac{\partial P}{\partial \bar{x}}\right)-\frac{C_{3 x} \bar{z}}{K_{o}}-\frac{2}{5} \frac{\bar{q}_{x}}{P}+C_{4 x}$,

$\bar{m}_{x z z}=\frac{16}{15} \frac{K_{o}}{P}\left(\varepsilon \frac{\partial P}{\partial \bar{x}}\right)$

and

$\bar{R}_{x z}=\frac{4 \sqrt{5}}{5}\left(C_{1 x} e^{-a \bar{z}}-C_{2 x} e^{a \bar{z}}\right)$.

The exponential parameter, $a=\sqrt{5} P /\left(3 K_{o}\right)$, controls the Knudsen layer width close to the wall. The integration constants $C_{1 x}, C_{2 x}, C_{3 x}$ and $C_{4 x}$ are determined from the wall boundary conditions given by Eqs. (31) and (32) with $n_{x}=n_{y}=0, n_{z}=1, \tau_{x}=1$, and $\tau_{y}=\tau_{z}=0$ for the bottom plate, located at $\bar{z}=0$, and for the slider located at $\bar{z}=h$ we have $n_{x}=-\sin \theta \simeq 0, n_{y}=0, n_{z}=-\cos \theta \simeq-1$, $\tau_{x}=\cos \theta \simeq 1, \tau_{y}=0$ and $\tau_{z}=-\sin \theta \simeq 0$. These terms can be expressed through the pressure gradient and bearing number, $\Lambda$, as:

$$
\begin{aligned}
& C_{1 x}=-\varepsilon \frac{\partial P}{\partial \bar{x}} C_{1}^{p}-\frac{\varepsilon \Lambda}{6 K_{o}} C_{1}^{u}, \\
& C_{2 x}=-\varepsilon \frac{\partial P}{\partial \bar{x}} C_{2}^{p}-\frac{\varepsilon \Lambda}{6 K_{o}} C_{2}^{u}, \\
& C_{3 x}=\varepsilon \frac{\partial P}{\partial \bar{x}} \frac{h}{2}+\frac{\varepsilon \Lambda}{K_{o}} \frac{D_{2}}{D_{1}}
\end{aligned}
$$

and 


$$
\begin{aligned}
C_{4 x}= & -\varepsilon \frac{\partial P}{\partial \bar{x}}\left[C_{4}^{p}+\left(\frac{h \gamma}{2}+\frac{7}{30} \frac{K_{o}}{P}\right) \frac{1}{P}\right] \\
& -\frac{\varepsilon \Lambda}{6 K_{o}}\left(C_{4}^{u}-1+\frac{6 \gamma D_{2}}{P D_{1}}\right) .
\end{aligned}
$$

The bearing number, $\Lambda$, is given by

$$
\Lambda=\frac{6 \mu u_{w} L}{P_{0} H_{0}^{2}}=\frac{6 \bar{u}_{w} K n}{\varepsilon} \sqrt{\frac{2}{\pi}}=\frac{6 \bar{u}_{w}}{\varepsilon} K_{o} .
$$

The solution for Eqs. (25)-(29) are:

$$
\begin{aligned}
& \bar{q}_{y}=C_{1 y} e^{-a \bar{z}}+C_{2 y} e^{a \bar{z}}+\frac{3}{2} \frac{K_{o}}{P}\left(\varepsilon \frac{\partial P}{\partial \bar{y}}\right), \\
& \bar{\sigma}_{y z}=-\left(\varepsilon \frac{\partial P}{\partial \bar{y}}\right) \bar{z}+C_{3 y}, \\
& \bar{v}=\frac{\bar{z}^{2}}{2 K_{o}}\left(\varepsilon \frac{\partial P}{\partial \bar{y}}\right)-\frac{C_{3 y} \bar{z}}{K_{o}}-\frac{2}{5} \frac{\bar{q}_{y}}{P}+C_{4 y},
\end{aligned}
$$

with the integration constants:

$$
\begin{aligned}
& C_{1 y}=-\varepsilon \frac{\partial P}{\partial \bar{y}} C_{1}^{p}, \\
& C_{2 y}=-\varepsilon \frac{\partial P}{\partial \bar{y}} C_{2}^{p}, \\
& C_{3 y}=\varepsilon \frac{\partial P}{\partial \bar{y}} \frac{h}{2}
\end{aligned}
$$

and

$C_{4 y}=-\varepsilon \frac{\partial P}{\partial \bar{x}}\left[C_{4}^{p}+\left(\frac{h \gamma}{2}+\frac{7}{30} \frac{K_{o}}{P}\right) \frac{1}{P}\right]$.

Expressions for the integration constants $C_{1}^{p}, C_{2}^{p}, C_{4}^{p}, C_{1}^{u}, C_{2}^{u}$ and $C_{4}^{u}$ arising in Eqs. (40)-(43) and (48)-(51) are listed in "Appendix".

Using the ideal gas law, $p=\rho R T$, the mass continuity Eq. (3) is rewritten as:

$\varepsilon \frac{\partial P}{\partial \bar{t}}+\frac{\partial P \bar{u}}{\partial \bar{x}}+\frac{\partial P \bar{v}}{\partial \bar{y}}+\frac{\partial P \bar{w}}{\partial \bar{z}}=0$.

Integrating Eq. (52) using the no-mass penetration condition given by Eq. (33) leads to a depth-averaged continuity equation:

$\varepsilon \frac{\partial P h}{\partial \bar{t}}+\frac{\partial}{\partial \bar{x}}\left[\int_{0}^{h} P \bar{u} \mathrm{~d} \bar{z}\right]+\frac{\partial}{\partial \bar{y}}\left[\int_{0}^{h} P \bar{v} \mathrm{~d} \bar{z}\right]=0$.
If we substitute Eqs. (37) and (47) into Eq. (53), we obtain the governing equation for pressure within the slider bearing as:

$$
\begin{gathered}
\frac{\partial P h}{\partial \bar{t}}+\frac{\partial}{\partial \bar{x}}\left\{\left[F_{P}-\left(\frac{P}{6 K_{o}}+\frac{\gamma}{h}\right) \frac{h^{3}}{2}\right] \frac{\partial P}{\partial \bar{x}}\right\} \\
+\frac{\partial}{\partial \bar{y}}\left\{\left[F_{P}-\left(\frac{P}{6 K_{o}}+\frac{\gamma}{h}\right) \frac{h^{3}}{2}\right] \frac{\partial P}{\partial \bar{y}}\right\} \\
+\frac{\partial}{\partial \bar{x}}\left(F_{C}+\frac{P \Lambda h}{12 K_{o}}\right)=0,
\end{gathered}
$$

in which

$F_{P}=\frac{6 \sqrt{5}}{25} \frac{K_{o}}{P}\left[\left(1-e^{-a h}\right) C_{1}^{p}-\left(1-e^{a h}\right) C_{2}^{p}\right]-P h C_{4}^{p}-\frac{5}{6} \frac{h K_{o}}{P}$

and

$$
\begin{aligned}
F_{C}= & \frac{\sqrt{5}}{25} \frac{\Lambda}{P}\left[\left(1-e^{-a h}\right) C_{1}^{u}-\left(1-e^{a h}\right) C_{2}^{u}\right] \\
& +\frac{P \Lambda h}{12 K_{o}}\left[1-2 C_{4}^{u}-\frac{6 D_{2}}{D_{1}}\left(\frac{2 \gamma}{P}+\frac{h}{K_{o}}\right)\right] .
\end{aligned}
$$

The underlined terms in Eq. (54) correspond to the NavierStokes solution with a first-order velocity-slip boundary, and the non-equilibrium behaviour introduced through the Knudsen layer is represented by $F_{P}$ and $F_{C}$ in Eqs. (55) and (56). It should be pointed that Eqs. (55) and (56) are merely algebraic expressions. In the Reynolds equation based on the Boltzmann equation (Fukui and Kaneko 1988; Cercignani et al. 2007), a significant number of terms are expressed in Abramowitz functions, which need to be evaluated accurately.

Equation (54) is a two-dimensional, time-dependent second-order partial differential equation, and it can be used to study the dynamic response of the gas between two walls. The numerical and computational effort in solving the extended Reynolds equations is negligible in comparison with the DSMC method. The DSMC approach needs to simulate the whole geometry of the slider bearing, while the extended Reynolds equation is one dimension less than the geometry. Furthermore, the DSMC method requires a sufficient number of particles to travel and collide for a certain time to ensure statistical accuracy. For example, it takes hours to simulate the DSMC validation cases referenced (Liu and $\mathrm{Ng} 2001$ ) in the next section, but it takes less than one second for the extended Reynolds equation to obtain the solution. In practical time-dependent engineering applications, the computational advantage of the present approach over the DSMC is very significant. 
In the next section, this equation will be validated against available DSMC data for slider bearing configurations. However, its application is beyond that. For example, it can readily be applied to study squeeze film air damping in MEMS (Bao and Yang 2007).

\section{Results and discussions}

Knowledge of the pressure distribution within the slider bearing is essential for bearing design. The DSMC data of Alexander, Garcia and Alder (1994) and Liu and Ng (2001) are used to evaluate the accuracy of the extended Reynolds Eq. (54) obtained from the R13 moment equations. In their DSMC studies, a steady two-dimensional slider bearing configuration was considered. For the simulations, the length of the channel was $L=5 \mu \mathrm{m}$ and the minimum channel height was fixed at, $H_{0}=50 \mathrm{~nm}$, which results in an aspect ratio $\varepsilon=H_{0} / L=0.01$. The angle of inclination, $\theta$, varied from 0.002 to $0.016 \mathrm{rad}$, so that the entrance channel height, $H_{1}$, ranges from 60 to $130 \mathrm{~nm}$. The working fluid in the DSMC study was argon gas with viscosity, $\mu=2.08 \times 10^{-5} \mathrm{~Pa} \mathrm{~s}$ and ambient conditions set to be $T=273 \mathrm{~K}$ and $p_{0}=1 \mathrm{~atm}(101,325 \mathrm{~Pa})$. This gives the Knudsen number, $K n$, a value of 1.24. The walls are treated as fully diffusive, i.e. $\alpha=1$. In the simulation of Alexander et al. (1994), hard sphere particles with a diameter of $3.66 \times 10^{-10} \mathrm{~m}$ were used, while in the study of Liu and $\mathrm{Ng}$ (2001), a variable hard sphere collision model with a diameter of $4.17 \times 10^{-10} \mathrm{~m}$ was employed.

The extended Reynolds Eq. (54) for two-dimensional steady-state flows can be reduced to:

$$
\begin{gathered}
\frac{\partial}{\partial \bar{x}}\left\{\left[F_{P}-\left(\frac{P}{6 K_{o}}+\frac{\gamma}{h}\right) \frac{h^{3}}{2}\right] \frac{\partial P}{\partial \bar{x}}\right\} \\
+\frac{\partial}{\partial \bar{x}}\left(F_{C}+\frac{P \Lambda h}{12 K_{o}}\right)=0 .
\end{gathered}
$$

Equation (57) can be solved numerically by the finite difference method, and we used 100 grid points uniformly across the slider bearing length. It takes less than a second using a personal computer. The computed pressure distribution obtained from Eq. (57) with two different molecular collision models is shown in Fig. 2 and compared with the DSMC data (Alexander et al. 1994; Liu and $\mathrm{Ng}$ 2001) for the case of $u_{w}=25 \mathrm{~m} / \mathrm{s}$, a bearing number, $\Lambda=61.6$, and pitch angle, $\theta=0.01 \mathrm{rad}$. As the Maxwell molecules and hard sphere model have similar collision constants, their results are close to each other. In addition, the pressure distributions for the MM and HS models are in good agreement with the DSMC data, although they are closer to the data of Alexander et al. (1994) than the results of Liu and $\mathrm{Ng}$ (2001). The discrepancy between the two sets of DSMC

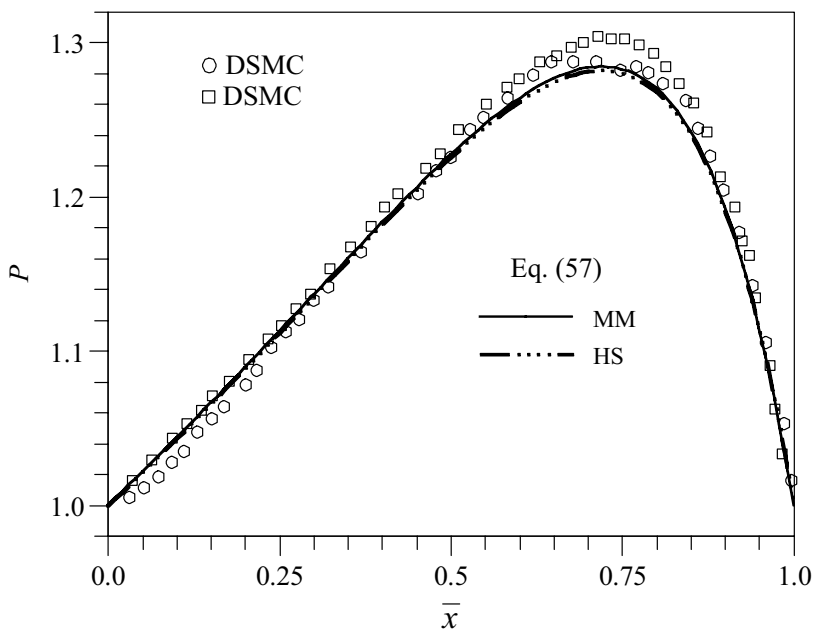

Fig. 2 Computed pressure distribution in the slider bearing for $K n=1.24, \Lambda=61.6$ and $\theta=0.01 \mathrm{rad}$. Lines: extended Reynolds Eq. (57) with MM and HS models. Symbols: DSMC data (circle Alexander et al. 1994; square Liu and Ng 2001)

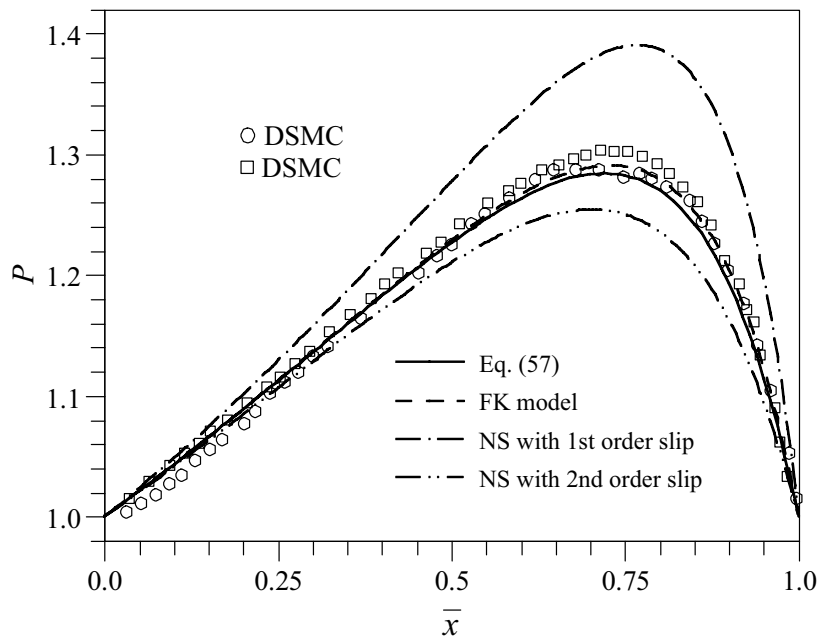

Fig. 3 Computed pressure distribution in the slider bearing for $K n=1.24, \Lambda=61.6$ and $\theta=0.01 \mathrm{rad}$. Symbols: DSMC data (circle Alexander et al. 1994; square Liu and $\mathrm{Ng} 2001$ )

data is mainly due to different particle size and collision models employed. Based on Fig. 2, only the results using the extended Reynolds equation with the MM model will be presented in the following sections.

The improvement of the extended Reynolds equation over the conventional Reynolds equation derived with a velocity-slip boundary condition is presented in Fig. 3. It is clear that the first-order slip boundary condition overpredicts the pressure rise, while the second-order boundary condition under-predicts the pressure change. The discrepancies between the conventional Reynolds equation with slip models and the DSMC data are so significant that they 
are unacceptable for engineering analysis. The extended Reynolds equation based on the R13 equations is also in good agreement with the FK model derived from the linearised Boltzmann equation (Fukui and Kaneko 1988; 1990), and both results are in close agreement with the data of Alexander et al. (1994).

\subsection{Effect of pitch angle $\theta$}

During its operation, the slider floats above the disk with an averaged pitching angle. The dynamic response of the pressure distribution to the variation of $\theta$ can be readily computed from Eq. (54). However, it is not easy to perform DSMC simulations with dynamic variation of $\theta$. Instead, a series of steady-state flow cases with different pitching angles were simulated with DSMC (Liu and $\mathrm{Ng} 2001$ ). The pressure distributions predicted by Eq. (57) in the slider bearing with $K n=1.24, \Lambda=61.6$ and $\theta=0.002$, $0.006,0.01$ and $0.016 \mathrm{rad}$, respectively, are presented in Fig. 4 and compared with the DSMC data (Liu and $\mathrm{Ng}$ 2001). At a low pitching angle, $\theta=0.002$ and $0.006 \mathrm{rad}$, the extended Reynolds equation reproduces the DSMC data fairly accurately. When the pitching angle is increased to $\theta=0.016 \mathrm{rad}$, Eq. (57) underestimates the peak pressure by about $1.5 \%$. As we continue to increase the value of $\theta$, the assumptions that underpin lubrication theory start to break down and the accuracy of the predictions using the extended Reynolds equation begin to deteriorate.

The pitching angle, $\theta$, influences the load capacity of the slider bearing, which is defined by the bearing force, $W$, as

$W=\frac{1}{L} \int_{0}^{L}\left(\frac{p-p_{0}}{p_{0}}\right) \mathrm{d} x=\int_{0}^{1}(P-1) \mathrm{d} \bar{x}$.

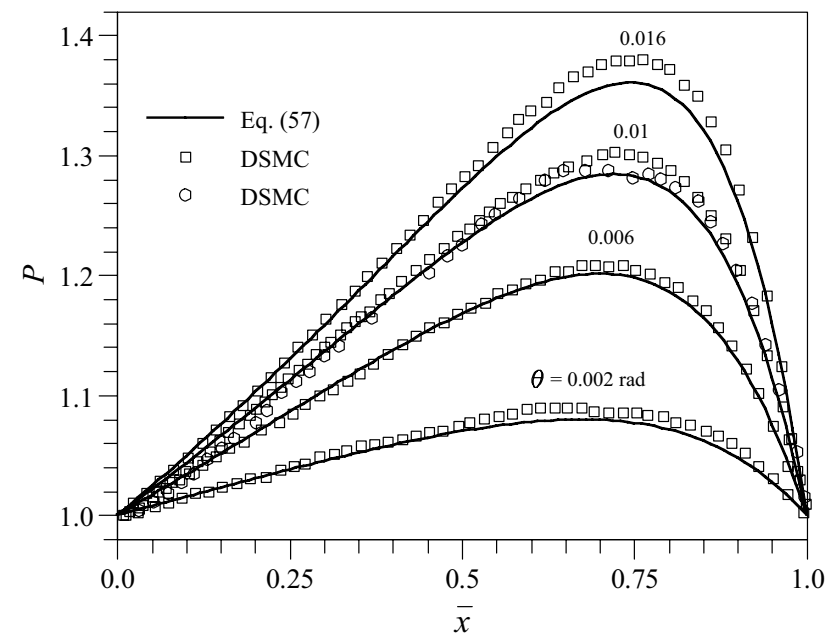

Fig. 4 Pressure distribution in the slider bearing for $K n=1.24$ and $\Lambda=61.6$ with different pitching angles, $\theta$. Symbols: DSMC (circle Alexander et al. 1994; square Liu and Ng 2001)

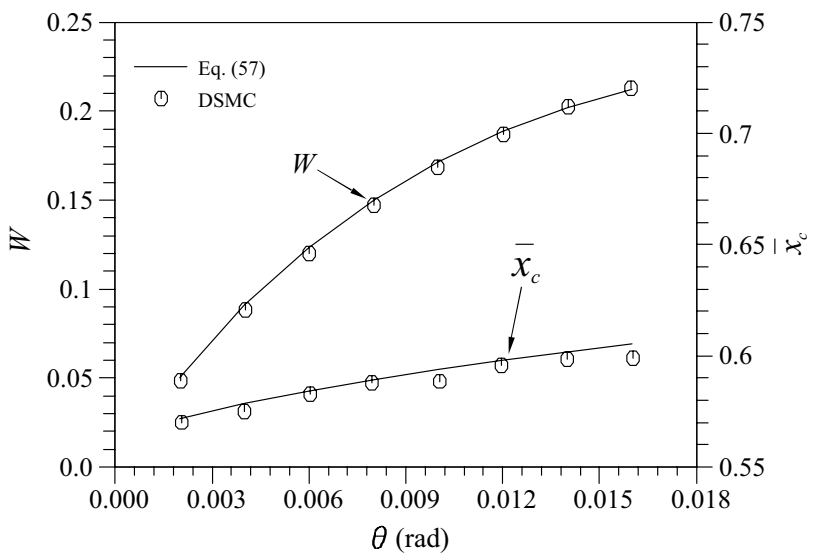

Fig. 5 Effect of the pitching angle $\theta$ on the bearing load capacity $W$ and load centre $\bar{x}_{c}$ in the slider bearing for $K n=1.24$ and $\Lambda=61.6$. Solid lines: extended Reynolds Eq. (57). Symbols: DSMC (Liu and $\mathrm{Ng}$ 2001)

The predicted bearing force, $W$, from the solution of the extended Reynolds equation, plotted against pitch angle, $\theta$, is shown in Fig. 5 and compared with available DSMC data (Liu and Ng 2001). Agreement is good, and both results indicate that as the value of $\theta$ increases, the value of $W$ will increase, which is consistent with the trends in Fig. 4. The load centre, $\bar{x}_{c}$, is the focal point of the resultant pressure on the slider surface, and it can be calculated from the pressure distribution by

$\bar{x}_{c}=\frac{\int_{0}^{1}(P-1) \bar{x} \mathrm{~d} \bar{x}}{\int_{0}^{1}(P-1) \mathrm{d} \bar{x}}$.

The position of the load centre moves towards the narrow end of the slider bearing as the pitch angle increases, as indicated in Fig. 5. However, the change of the centre position is only slight.

\subsection{Effect of bearing number $\Lambda$ and Knudsen number Kn}

It can be seen from Eqs. (54) or (57) that the bearing number, $\Lambda$, is one of the important parameters and can influence the pressure distribution significantly. For a fixed value of $K n=1.24$ and $\theta=0.01 \mathrm{rad}$, the DSMC data of three different bearing numbers are available to validate the extended Reynolds equation. Liu and Ng (2001) simulated the operating conditions of low and medium bearing numbers, $\Lambda=61.6,123.2$, and 221.8 , respectively. The predicted pressure distributions from the extended Reynolds equation agree with the DSMC data quite well. For a high bearing number, $\Lambda=758$, the predicted pressure distribution from the macroscopic equation is in excellent agreement with the DSMC data of Alexander, Garcia and Alder 
(1994). In this case, the corresponding Mach number is 1.0, yet the extended Reynolds equation still performs well.

The pressure distributions at different values of $K n$ for a slider bearing with $\Lambda=61.6$ and $\theta=0.01 \mathrm{rad}$ are shown in Fig. 7. At a transitional Knudsen number of 0.5, the bearing generates more than a $55 \%$ pressure rise. The pressure profile predicted by the extended Reynolds equation is close to the FK model $(1988,1990)$ with a slight over-prediction of the peak pressure. As the Knudsen number increases, with the bearing number fixed, the disk velocity is reduced so that the magnitude of the pressure rise reduces. At $K n=1.24$, the pressure rise is reduced by about $30 \%$. The FK model (1988, 1990), DSMC data (Alexander et al. 1994), and Eq. (57) are all in good agreement. As the Knudsen number increases further, the extended Reynolds equation gradually starts to under-predict the pressure rise predicted by the FK model. This is due to the fact that a set of 13 moments is not sufficient to predict correctly the flow rate for Poiseuille flow at such large Knudsen numbers (Struchtrup and Torrilhon 2008; Gu and Emerson 2009). It should be noted that the pressure in the extended Reynolds equation is an averaged quantity across the gap. The good agreement of pressure between the DSMC data and the extended Reynolds equation at a Knudsen number around unity does not imply the R13 equations can accurately predict the flow field but they will provide qualitatively reliable information.

Comparing Figs. 6 and 7 indicates that $\Lambda$ and $K n$ have opposite effects on the pressure rise, which will affect the load capacity of the slider bearing. For a slider bearing with $\theta=0.01 \mathrm{rad}$ and $\varepsilon=0.01$, the bearing force diagram constructed from the solution of Eq. (57) is shown in Fig. 8 for different values of $K n$ and $\Lambda$. For a fixed value of $\Lambda$, the bearing force, $W$, decreases as the Knudsen number increases. The lower the bearing number, the more rapidly the bearing force drops as $K n$ increases. For a fixed Knudsen number, the bearing force increases as the bearing number increases.

\subsection{Predicted velocity profiles and velocity at the wall}

Using the extended Reynolds equation, the velocity profile between the moving disk and the fixed slider can be calculated from Eq. (37). It is worth noting that the tangential heat flux, $q_{x}$, is involved in Eq. (37), even if the conditions are assumed to be isothermal. This non-equilibrium effect is clearly captured by the exponentials in Eq. (35) and contributes to the characteristic Knudsen layer description. The predicted velocity profiles from Eq. (37) at $\bar{x}=0,0.5$, and 1 are shown in Fig. 9 for a slider bearing with $\varepsilon=0.01$, $K n=1.24$ and $\Lambda=61.6$. In general, the macroscopic predictions qualitatively follow the DSMC data (Liu and $\mathrm{Ng}$ 2001) at the three locations, although they cannot capture

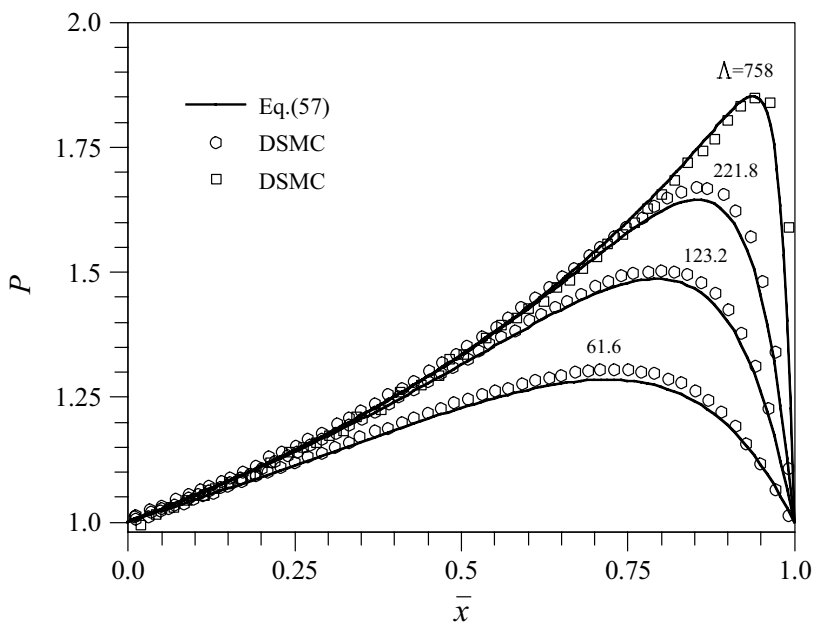

Fig. 6 Effect of the bearing number, $\Lambda$, on the pressure distribution in the slider bearing for $K n=1.24$ and $\theta=0.01$ rad. Solid lines: extended Reynolds Eq. (57). Symbols: DSMC (circle Alexander et al. 1994; square Liu and $\mathrm{Ng} 2001$ )

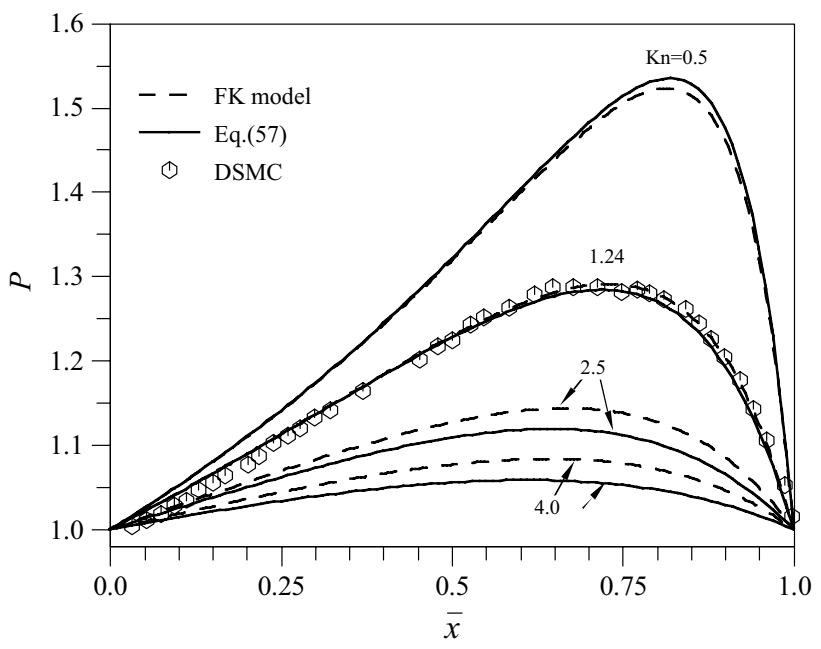

Fig. 7 Effect of the Knudsen number $K n$ on the pressure distribution for the slider bearing with $\Lambda=61.6$ and $\theta=0.01 \mathrm{rad}$. Symbols: DSMC (Alexander et al. 1994)

the Knudsen layer accurately. As indicated in a previous study of Kramers problem (Gu et al. 2009, 2010), higherorder moment equations, such the R26 equations ( $\mathrm{Gu}$ and Emerson 2009), are necessary to describe the Knudsen layer effect accurately. Nevertheless, the present extended Reynolds equation and its associated velocity expression are useful in bearing analysis and design.

The velocity predicted for the walls indicate a higher level of slip on both the static upper wall and the moving lower wall, as shown in Fig. 9. Figure 10 presents the predicted normalised slip velocity from the macroscopic model on the bottom wall for three different bearing numbers for 


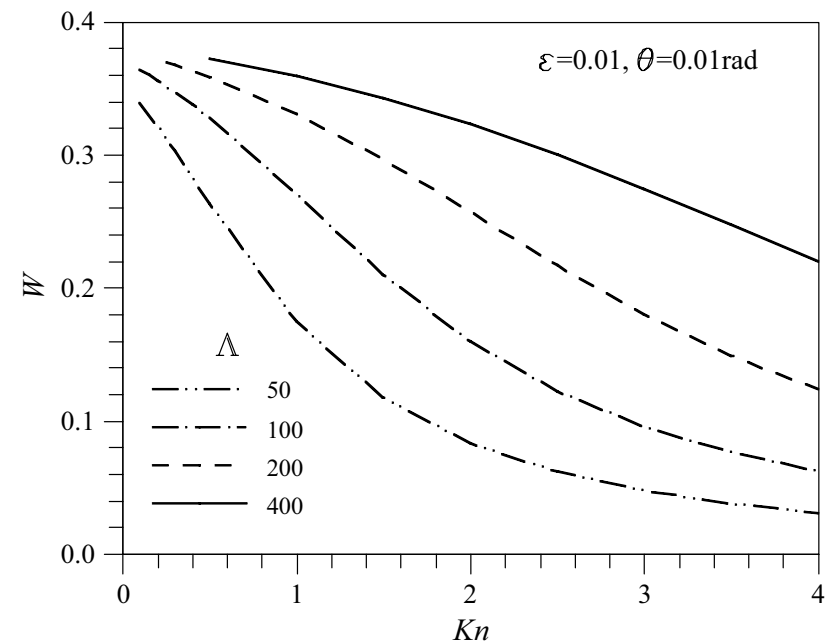

Fig. 8 Effects of the Knudsen number $K n$ and the bearing number $\Lambda$ on the bearing force for a slider bearing with $\varepsilon=0.01$ and $\theta=0.01$ $\mathrm{rad}$

a slider bearing with $\varepsilon=0.01, \theta=0.01 \mathrm{rad}$ and $K n=1.24$, in comparison with DSMC data (Liu and Ng 2001). It can be seen that the velocity slip is over-predicted for all three cases, particularly for the case with a low bearing number, $\Lambda=61.6$. The over-prediction of the slip velocity is related to the poor description of the Knudsen layer close to the wall. As the bearing number increases, the prediction of the slip velocity gradually begins to improve.

\subsection{Shear stress on the moving lower wall}

The shear stress on the wall can be as important as the pressure distribution in the design of a slider bearing. In the present approach, the shear stress can be calculated from Eq. (36) once the solution of the extended Reynolds equation has been obtained. An extensive DSMC study of the shear stress on the lower wall was carried out by $\mathrm{Ng}$ et al. (2002). For a slider bearing with $K n=1.24$ and $\theta=0.01$ $\mathrm{rad}$, three aspect ratios $\varepsilon=0.005,0.01$ and 0.02 were set up with three wall velocities, $u_{w}=25,50$ and $100 \mathrm{~m} / \mathrm{s}$, respectively, to achieve a bearing number, $\Lambda=123.2$. The shear stress for the three cases is shown in Fig. 11, with the lines representing the solution from Eq. (36) and symbols the DSMC data. The agreement between the present study, using Eq. (36), and the DSMC data is good with the extended Reynolds equation predicting slightly higher values of stress. From the values of shear stress, the drag force on the walls can be calculated.
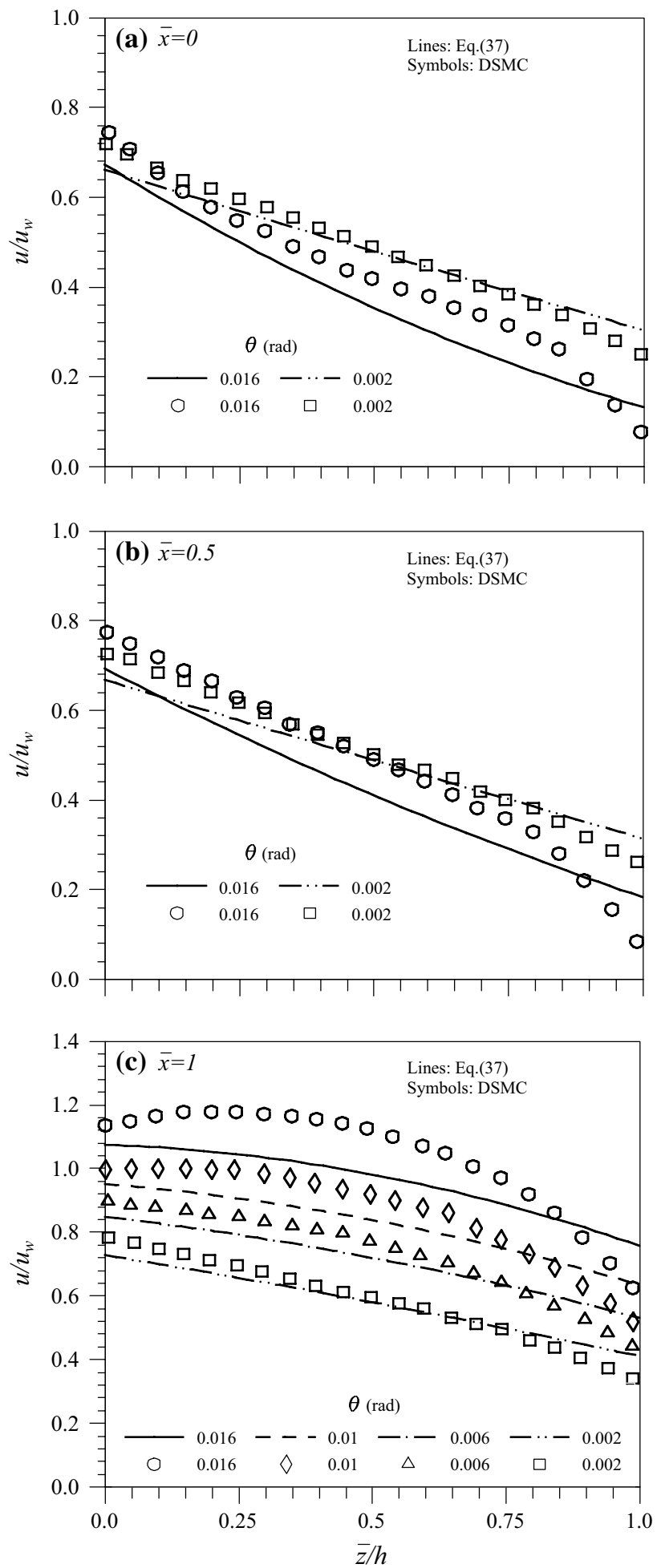

Fig. 9 Stream-wise velocity profiles at three different locations a $\bar{x}=0 ; \mathbf{b} \bar{x}=0.5 ; \mathbf{c} \bar{x}=1$; for a slider bearing with $\varepsilon=0.01$, $K n=1.24$ and $\Lambda=61.6$. Lines: velocity Eq. (37). Symbols: DSMC (Liu and Ng 2001) 


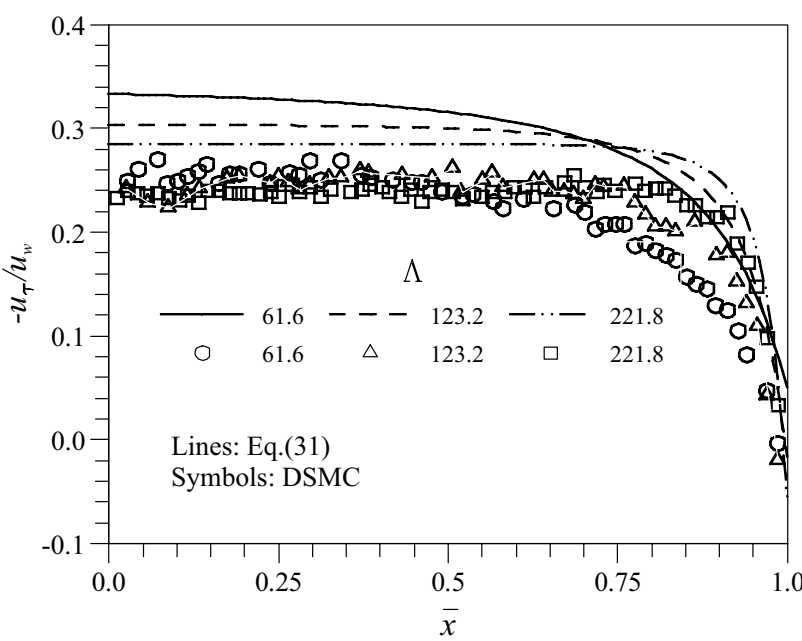

Fig. 10 Velocity slip on the lower wall for a slider bearing with $\varepsilon=0.01, \theta=0.01 \mathrm{rad}$ and $K n=1.24$. Lines: velocity slip Eq. (31). Symbols: DSMC (Liu and Ng 2001)

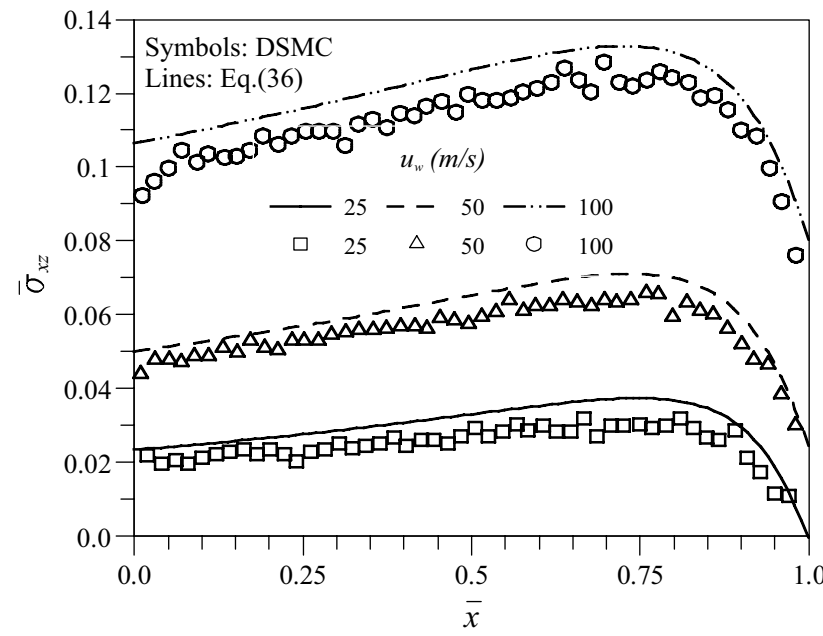

Fig. 11 Shear stress on the lower wall for a slider bearing with $\Lambda=123.2, \theta=0.01 \mathrm{rad}, K n=1.24$ and three different values of $\varepsilon$. Lines: stress Eq. (36). Symbols: DSMC data (Ng et al. 2002)

\section{Conclusions}

Using lubrication theory, we have derived an extended Reynolds equation based on the regularised 13 moment equations. This new set of equations is able to capture all of the important non-equilibrium effects associated with gaseous transport between narrow gaps and can readily be used in the design of new slider bearings. Results obtained with this new set of equations have been compared with available direct simulation Monte Carlo data and are shown to be significantly better than current Navier-Stokes models that incorporate velocity slip. Moreover, the equations are also able to predict shear stress distributions and velocity fields, although the latter indicates that resolution of the Knudsen layer is restricted with the 13 moment equations and a higher-order set of equations is necessary if a more detailed analysis of the Knudsen layer is required. In general, however, this new set of equations performs very well, is straightforward to implement for robust engineering design, and will provide a useful advance in modelling gaseous transport in small gaps.

\section{Appendix}

The terms in the integration constants of Eqs. (40)-(43) and (48)-(51) can be written as:

$C_{1}^{p}=2\left[\sqrt{5} \gamma\left(e^{a h}+1\right)+3\left(e^{a h}-1\right)\right] D_{3}$,

$C_{2}^{p}=2\left[\sqrt{5} \gamma\left(e^{-a h}+1\right)-3\left(e^{-a h}-1\right)\right] D_{3}$,

$C_{4}^{p}=\frac{4[\sqrt{5} \gamma(\cosh (a h)+1)+3 \sinh (a h)] D_{3}}{5 P}$,

$C_{1}^{u}=\frac{10 \gamma\left[\sqrt{5} \gamma\left(e^{a h}-1\right)+3\left(e^{a h}+1\right)\right]}{3 D_{1}}$,

$C_{2}^{u}=\frac{10 \gamma\left[\sqrt{5} \gamma\left(e^{-a h}-1\right)-3\left(e^{-a h}+1\right)\right]}{3 D_{1}}$,

$C_{4}^{u}=\frac{4 \gamma[\sqrt{5} \gamma(\cosh (a h)-1)+3 \sinh (a h)]}{3 P D_{1}}$,

in which,

$$
\begin{aligned}
D_{1}= & 6 D_{2}\left(\frac{h}{K_{o}}+\frac{2 \gamma}{P}\right) \\
& +\frac{8}{3} \frac{\gamma}{P}[\sqrt{5} \gamma(\cosh (a h)-1)+3 \sinh (a h)],
\end{aligned}
$$

$D_{2}=\frac{1}{9}\left[\left(81+20 \gamma^{2}\right) \sinh (a h)+48 \sqrt{5} \gamma \cosh (a h)\right]$,

and

$D_{3}=\frac{5}{9 D_{2}}\left(\frac{\gamma h}{4}+\frac{7 K_{o}}{3 P}\right)$.

Acknowledgments XJG and DRE would like to express their gratitude to the UK Engineering and Physical Sciences Research Council (EPSRC) for their support under Collaborative Computational Project 12 (CCP12) and Programme Grant (EP/I011927/1). HZ appreciates 
the financial support from the National Natural Science Foundation of China (Grant No. 11102071) and China Scholarship Council (Grant No.201208330295) and would like to thank STFC Daresbury Laboratory and Professor Y.H. Zhang in University of Strathclyde to host his academic visit in UK.

Open Access This article is distributed under the terms of the Creative Commons Attribution 4.0 International License (http://creativecommons.org/licenses/by/4.0/), which permits unrestricted use, distribution, and reproduction in any medium, provided you give appropriate credit to the original author(s) and the source, provide a link to the Creative Commons license, and indicate if changes were made.

\section{References}

Abramowitz M, Stegun I (1970) Handbook of mathematical functions. Dover Publications, New York

Alexander FJ, Garcia AJ, Alder BJ (1994) Direct simulation Monte Carlo for thin-film bearings. Phys Fluids 6:3854-3860

Bahukudumbi P, Beskok A (2003) A phenomenological lubrication model for the entire Knudsen regime. J Micromech Microeng 13:873-884

Bao M, Yang H (2007) Squeeze film air damping in MEMS. Sens Actuators, A 136:3-27

Barber RW, Emerson DR (2006) Challenges in modelling gas-phase flow in microchannels: from slip to transition. Heat Transfer Eng 27:3-12

Bhatnagar PL, Gross EP, Krook M (1954) A model for collision processes in gases. I. Small amplitude processes in charged and neutral one-component systems. Phys Rev 94:511-525

Burgdorfer A (1959) The influence of the molecular mean free path on the performance of hydrodynamic gas lubricated bearings. ASME J Basic Eng 81:94-100

Cercignani C (2000) Rarefied gas dynamics: from basic concepts to actual calculations. Cambridge University Press, Cambridge

Cercignani C, Lampis M, Lorenzani S (2004) Variational approach to gas flows in microchannels. Phys Fluids 16:3426-3437

Cercignani C, Lampis M, Lorenzani S (2007) On the Reynolds equation for linearized models of the Boltzmann operator. Transp Theory Stat Phys 36:257-280

Chen D, Bogy DB (2010) Comparisons of slip-corrected Reynolds lubrication equations for the air bearing film in the head-disk interface of hard disk drives. Tribol Lett 37:191-201

Fukui S, Kaneko R (1988) Analysis of ultra-thin gas film lubrication based on linearized Boltzmann equation: first report-derivation of a generalized lubrication equation including thermal creep flow. ASME J Tribol 110:253-262

Fukui S, Kaneko R (1990) A database for interpolation of Poiseuille flow rates for high Knudsen number lubrication problems. ASME J Tribol 112:78-83

Grad H (1949) On the kinetic theory of rarefied gases. Commun Pure Appl Math 2:331-407

Gu XJ, Emerson DR (2007) A computational strategy for the regularized 13 moment equations with enhanced wall-boundary conditions. J Comput Phys 225:263-283

Gu XJ, Emerson DR (2009) A high-order moment approach for capturing non-equilibrium phenomena in the transition regime. $\mathrm{J}$ Fluid Mech 636:177-216
Gu XJ, Emerson DR (2014) Linearized-moment analysis of the temperature jump and temperature defect in the Knudsen layer of a rarefied gas. Phys Rev E 89:063020

Gu XJ, Emerson DR, Tang GH (2009) Kramers' problem and the Knudsen minimum: a theoretical analysis using a linearised 26-moment approach. Continuum Mech Thermodyn 21:345-360

Gu XJ, Emerson DR, Tang GH (2010) Analysis of the slip coefficient and defect velocity in the Knudsen layer of a rarefied gas using the linearized moment equations. Phys Rev E 81:016313

Gupta V, Torrilhon M (2012) Automated Boltzmann collision integrals for moment equations. In: Proceedings of the 28th international symposium on rarefied gas dynamics, AIP conference proceeding 1501, pp 67-74

Hsia YT, Domoto GA (1983) An experimental investigation of molecular rarefaction effects in gas lubricated bearings at ultra-low clearance. ASME J Tribol 105:120-129

Jiang JZ, Shen C, Fan J (2005) Statistical simulation of thin-film bearings. In: Proceedings of the 24th international symposium on rarefied gas dynamics, AIP conference proceeding, pp 180-185, $762 \mathrm{pp}$

John B, Damodaran M (2009) Computation of head-disk interface gap micro flowfields using DSMC and continuum-atomistic hybrid methods. Int J Numer Meth Fluids 61:1273-1298

Lilley CR, Sader JE (2008) Velocity profile in the Knudsen layer according to the Boltzmann equation. Proc R Soc A 464:2015-2035

Liu N, Ng EY-K (2001) The posture effects of a slider air bearing on its performance with a direct simulation Monte Carlo method. J Micromech Microeng 11:463-473

Maxwell JC (1879) On stresses in rarified gases arising from inequalities of temperature. Philos Trans R Soc Lond 170:231-256

Mitsuya Y (1993) Modified Reynolds equation for ultra-thin film gas lubrication using 1.5-order slip-flow model and considering surface accommodation coefficient. ASME J Tribol 115:289-294

Ng EY-K, Liu N, Mao X (2002) Stress-density ratio slip-corrected Reynolds equation for ultra-thin film gas bearing lubrication. Phys Fluids 14:1450-1457

Reynolds O (1886) On the theory of lubrication and its application to Mr. Beauchamp Tower's experiments, including an experimental determination of the viscosity of olive oil. Proc R Soc Lond 177:157-234

Struchtrup H (2005) Macroscopic transport equations for rarefied gas flows. Springer, Berlin

Struchtrup H (2008) Linear kinetic transfer: moment equations, boundary conditions, and Knudsen layer. Phys A 387:1750-1766

Struchtrup H, Torrilhon M (2003) Regularization of Grad's 13 moment equations: derivation and linear analysis. Phys Fluids $15: 2668-2680$

Struchtrup H, Torrilhon M (2008) Higher-order effects in rarefied channel flows. Phys Rev E 78:046301

Torrilhon M, Struchtrup H (2008) Boundary conditions for regularized 13-moment-equations for micro-channel-flows. J Comput Phys 227:1982-2011

Yang Q, Zhang H, Liu Y (2015) Improved modified Reynolds equation for thin-film gas lubrication from an extended slip velocity boundary condition. Microsyst Technol. doi:10.1007/ s00542-015-2667-4 